\title{
Regeneration after acute kidney injury requires PTIP-mediated epigenetic modifications
}

\author{
Abdul Soofi, Ana P. Kutschat, Mohammad Azam, Ann M. Laszczyk, and Gregory R. Dressler \\ Department of Pathology, Michigan Medicine, University of Michigan, Ann Arbor, Michigan, USA.
}

\begin{abstract}
A terminally differentiated cellular phenotype is thought to be maintained, at least in part, by both active and repressive histone marks. However, it is unclear whether regenerating cells after injury need to replicate such epigenetic marks to recover. To test whether renal epithelial cell regeneration is dependent on histone H3K4 methylation, we generated a mouse model that deleted the Paxip1 gene in mature renal proximal tubules. Paxip1 encodes PTIP, an essential protein in the MII3/4 histone H3K4 methyltransferase complex. Mice with PTIP deletions in the adult kidney proximal tubules were viable and fertile. Upon acute kidney injury, such mice failed to regenerate damaged tubules, leading to scarring and interstitial fibrosis. The inability to repair damage was likely due to a failure to reenter mitosis and reactivate regulatory genes such as Sox9. PTIP deletion reduced histone H3K4 methylation in uninjured adult kidneys but did not significantly affect function or the expression of epithelial specific markers. Strikingly, cell lineage tracing revealed that surviving PTIP mutant cells could alter their phenotype and lose epithelial markers. These data demonstrate that PTIP and associated MLL3/4-mediated histone methylation are needed for regenerating proximal tubules and to maintain or reestablish the cellular epithelial phenotype.
\end{abstract}

Conflict of interest: The authors declare that no conflict of interest exists.

Copyright: () 2020, American Society for Clinical Investigation.

Submitted: May 13, 2019

Accepted: January 3, 2020

Published: February 13, 2020.

Reference information: /CI Insight. 2020;5(3):e130204.

https://doi.org/10.1172/jci.

insight.130204.

\section{Introduction}

Epigenetic modifications of the genome are thought to impart cell- and tissue-specific patterns of gene expression and, hence, cellular phenotypes through the specific methylation of histone tails and the methylation of DNA at CpG islands $(1,2)$. In development, histone modification correlates with the loss of pluripotency and the establishment of cell lineages, and it is thought to impart cellular memory whereby the epigenome of a progenitor cell provides a template for all subsequent derivatives $(3,4)$. Such histone modifications are imprinted by the Polycomb and Trithorax families of epigenetic regulators that mark the epigenome with silencing or activating histone modifications, respectively (5-7). Still, the questions remain as to how stable such epigenetic modifications are and whether alterations in the epigenome can impact the initiation or progression of disease. Such questions are especially relevant in long-lived cell types, which are not mitotically active but must be able to respond to injury in the event of tissue damage.

The proximal tubules of the adult kidney consist of stable, terminally differentiated epithelial cells that have exited the cell cycle. If they are renewed, it is at a very slow rate; few, if any, epithelial cells in a healthy adult kidney undergo cell division. However, in response to acute kidney injury (AKI) from ischemia or nephrotoxicity, the proximal tubule cells can regenerate damaged tubules $(8,9)$. The best available evidence suggests that there are no defined proximal tubule stem cells. Instead, the surviving epithelial cells can become mitotically active and repopulate the damaged tubules (10-12). This process of regeneration must require changes in gene expression of surviving epithelial cells, which may involve the reactivation of genes controlling development and proliferation.

Evidence suggests that the process of AKI and subsequent regeneration also requires alterations or resetting of the epigenome in proximal tubule cells. For example, loss of the polycystic kidney disease gene ADPK1 in nondividing renal epithelial cells does not lead to cyst formation until the kidney is subjected to acute injury $(13,14)$. Similarly, deletion of cilia in adult cells does not lead to cysts until acute injury is induced (15). The transcription factor HNF1 $\beta$ activates the PKD2 gene in the developing kidney but is not required for continued PKD2 expression in an adult epithelial cell (16), suggesting some degree of epigenetic memory in adult cells once the pattern of gene expression is set in development. However, upon AKI, 
HNF1 $\beta$ is again required to activate the PKD2 gene in regenerating proximal tubules. These data strongly suggest that regeneration of proximal tubule cells requires a resetting of epigenetic memory. However, few studies have examined the role of epigenetic writers in AKI more directly.

In order to more directly test the requirement for epigenetic mechanisms in kidney regeneration, we utilized a conditional allele of Paxip1, which encodes the adaptor protein PTIP (Pax transactivation-domain interacting protein). The PTIP protein is part of an MLL3/4 complex that imprints an active histone methylation mark at histone $\mathrm{H} 3$, lysine $4(\mathrm{H} 3 \mathrm{~K} 4)(17,18)$. Prior work has shown that deletion of PTIP in terminally differentiated cells can alter the gene expression patterns in podocytes (19) or cardiac myocytes $(20,21)$, but it does not lead to an immediate disease phenotype. In this report, we utilized a Pepck-Cre driver strain to delete PTIP in differentiated mouse proximal tubule cells. The kidneys of mice carrying the PTIP deletion appeared normal, with little evidence for loss of kidney function or other gross abnormalities. However, when such mice were subjected to AKI, the ability to repair and repopulate damaged tubules was severely compromised. These data show that de novo histone H3K4 methylation is necessary for differentiated cells to reenter mitosis and regenerate the kidney. These data suggest that the epigenome of a differentiated cell, while stable under normal conditions, must require new histone methylation to reset or replicate the epigenome once cells are stimulated to divide.

\section{Results}

Creating proximal tubule-specific PTIP deletions. Deletion of PTIP in more terminally differentiated cells, such as podocytes or cardiac myocytes, suggested that differentiated cells did not immediately require the M113/4 H3K4 methyltransferase activity for function. To test whether the PTIP-dependent H3K4 methylation was important for recovery from AKI, we utilized a conditional Paxip1-floxed allele and a Pepck-Cre driver strain to delete PTIP in a subset of proximal tubule epithelial cells. Some mice also carried the Gt(Rosa)26Sor ${ }^{\text {tm4(ACTB-tdTomato,-EGFP) Luo }}$ reporter allele, which expressed a membrane-bound Tomato red protein in all cells but switched to a membrane EGFP in cells that saw activated Cre recombinase (Supplemental Figure 1, A and B; supplemental material available online with this article; https://doi.org/10.1172/ jci.insight.130204DS1). The Pepck-Cre transgene is expressed late in nephrogenesis, when more mature proximal tubules are formed, and it is active in the S1, S2, and S3 segments of the nephron (22). Active Cre can be easily discerned by reporter EGFP expression in the developing cortex of a newborn mouse kidney but not in the nephrogenic zone, the medulla, or in the glomeruli (Supplemental Figure 1). In mice carrying 2 PTIP-floxed alleles (Paxip $1^{f l f f}$ ) and the Pepck-Cre transgene, PTIP protein was clearly reduced in lysates from the kidney cortex of adults but not in lysates from the medulla (Supplemental Figure 1, C and D). Similarly, immunostaining for PTIP protein in newborns shows a reduction of nuclear PTIP staining that is coincident with EGFP reporter expression in the Paxip $1^{f l / f l}$ Pepck-Cre (hereafter referred to as PTIP-) mice both in newborns and in adult kidney sections (Supplemental Figure 1, E-G). These data validate the specific deletion of PTIP in kidney proximal tubules.

Despite PTIP deletion, mice had no gross morphological phenotypes and remained healthy and fertile, suggesting that PTIP deletion at this stage of differentiation had little apparent affect on kidney development or function. Histology of adult kidneys from PTIP ${ }^{-}$and Paxip $1^{f l+}$ Pepck-Cre (or Paxip $1^{f l / f}$, hereafter termed PTIP ) mice appeared similar (Supplemental Figure 2), with no evidence of fibrosis or kidney injury, as evaluated by staining for $\alpha$-smooth muscle actin ( $\alpha$ SMA), Collagen IV, or kidney injury molecule 1 (Kim1). However, PTIPkidneys did show low levels of lipocalin-2/neutrophil gelatinase-associated lipocalin (Ngal) staining in tubules that were more distal from the GFP marked proximal tubules that had deleted PTIP. Lcn2 is an early marker for tubular injury $(23,24)$, suggesting that PTIP deletion in proximal tubules was not entirely benign.

PTIP mutant kidneys fail to regenerate after AKI. Adult mice with the proximal tubule PTIP deletion (PTIP ${ }^{-}$) and controls $\left(\mathrm{PTIP}^{+}\right)$were subjected to AKI by injection of a single i.p. dose of folic acid (FA). At a dose of $250 \mathrm{mg} / \mathrm{kg}$, a nephrotoxic response was rapidly induced, resulting in an increase in serum creatinine and blood urea nitrogen (BUN) by 48 hours and significant proteinuria at 24 hours after FA (Figure 1, A and B) in both $\mathrm{PTIP}^{-}$and $\mathrm{PTIP}^{+}$mice. We noted an increase in mortality among the PTIP cohort, with $51 \%$ mortality by 3 days after FA, compared with $12 \%$ for $\mathrm{PTIP}^{+}$controls. However, the surviving $\mathrm{PTIP}^{-}$mice recovered renal function over time, as did the $\mathrm{PTIP}^{+}$control mice (Figure $1 \mathrm{~B}$ ). During the acute phase, 2 days after FA, both PTIP $^{-}$and PTIP $^{+}$kidneys exhibited significant tubular dilation, expression of Kim 1 along the apical surfaces of dilated tubules, and increased $\alpha$ SMA-staining activated fibroblasts within the interstitium (Figure 1C). 


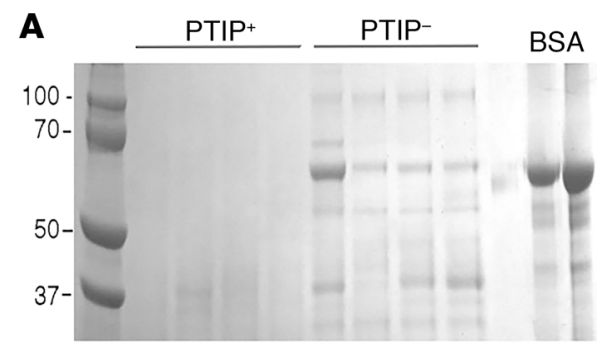

No injury
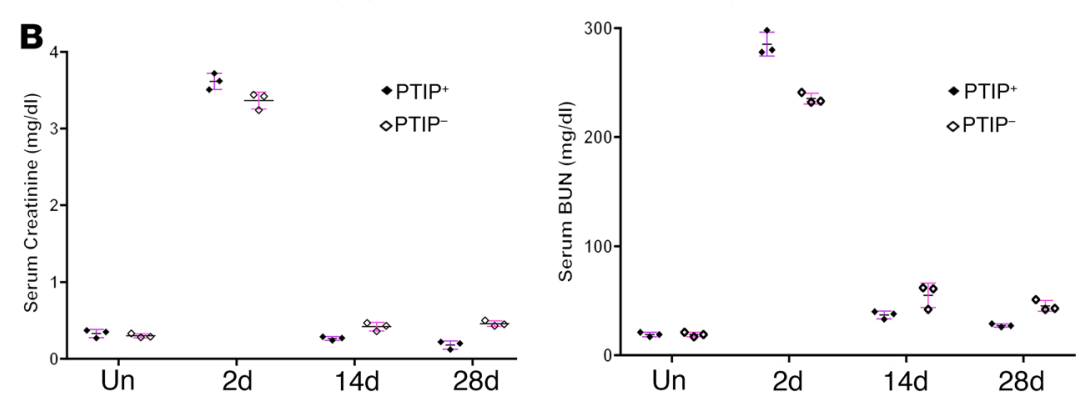

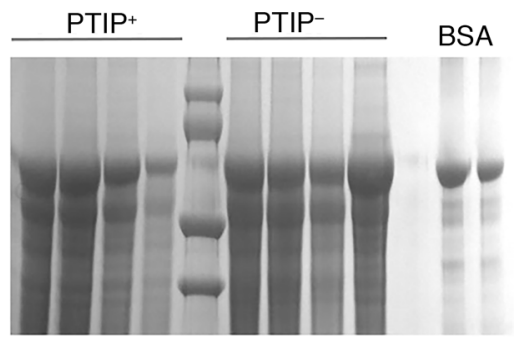

24h after AKI

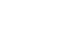

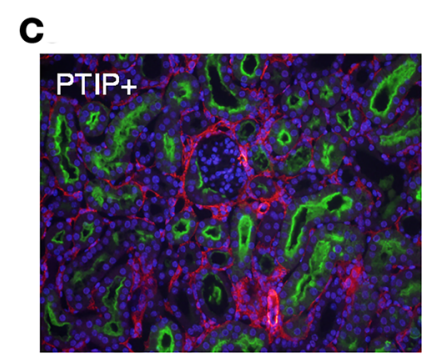

Kim1/aSMA

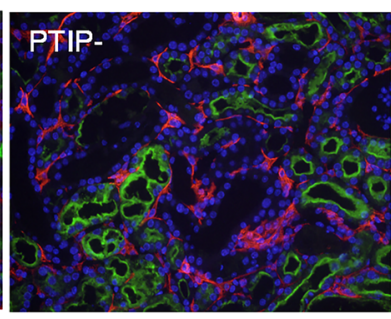

Kim1/aSMA

Figure 1. Acute kidney injury in PTIP- kidneys. Mice were given a single dose of folic acid and analyzed for renal function. (A) Proteinuria is strongly induced 24 hours after AKI in both PTIP+ and PTIP- mice, whereas PTIP- mice have a slightly higher baseline of protein in urine prior to injury. (B) Serum creatinine and blood urea nitrogen (BUN) in mice before injury or at indicated days after AKI. Note highly elevated creatinine and BUN at 2 days after folic acid. Mice that recover show no significant differences in creatinine or BUN by 14 and 28 days after AKI. For each time point and genotype, 3 separate animals were tested. (C) Immunostaining for Kim1 (green) and $\alpha$ SMA (red) indicated significant injury in PTIP+ and PTIP- kidneys 2 days after folic acid injection.

Among the surviving population, we examined the kidneys at 2, 7, 14, and 28 days after FA injections and assessed for damage and evidence of recovery. At 2 days after FA, both PTIP $^{-}$and PTIP $^{+}$kidneys exhibited increased $\alpha$ SMA staining-activated fibroblasts within the interstitium (Figure 2). By 7 days after AKI, the levels of $\alpha$ SMA staining began to decrease in PTIP $^{+}$kidneys yet remained high in the PTIPkidneys. By 14 and 28 days after AKI, the $\mathrm{PTIP}^{+}$kidneys had fully recovered, with little evidence of prior damage. However, the PTIP- kidneys had evidence of fibrosis and scarring, with areas denuded in tubules that were replaced by $\alpha \mathrm{SMA}^{+}$cells and extracellular matrix deposits (Figure 2). Given that the $\mathrm{PTIP}^{-}$mice did recover renal function after 14 days, as measured by creatinine clearance and BUN, this suggests that not all proximal tubules were damaged with enough remaining to provide sufficient creatinine clearance.

We characterized the recovery from AKI in more detail. Quantitation of total $\alpha$ SMA was done by Western blotting at 2, 7, 14, and 28 days after AKI and by immunostaining of kidney sections (Figure $3, \mathrm{~A}-\mathrm{C})$. These data reveal a significant increase in $\alpha \mathrm{SMA}$ total protein and interstitial area in the PTIPand $\mathrm{PTIP}^{+}$mice at 2 days after AKI. However, as $\mathrm{PTIP}^{+}$mice recovered, the $\alpha \mathrm{SMA}$ levels and interstitial areas decreased, whereas both metrics remained high in the PTIP- kidneys. Expression of Kim1 remained high along the apical surfaces of tubules at 7 days in both genotypes, but by 14 days, PTIP $^{+}$ kidneys showed little Kim1, whereas many PTIP- tubules continued to stain strongly for Kim1 (Figure 3D). Some residual Kim1 staining could be detected 28 days after AKI in PTIP $^{-}$kidneys but not in PTIP controls. Areas denuded in tubules in the PTIP- kidneys exhibited trichrome staining for cross-linked collagen, which was not observed in the $\mathrm{PTIP}^{+}$kidneys at 28 days (Figure $3 \mathrm{E}$ ). 


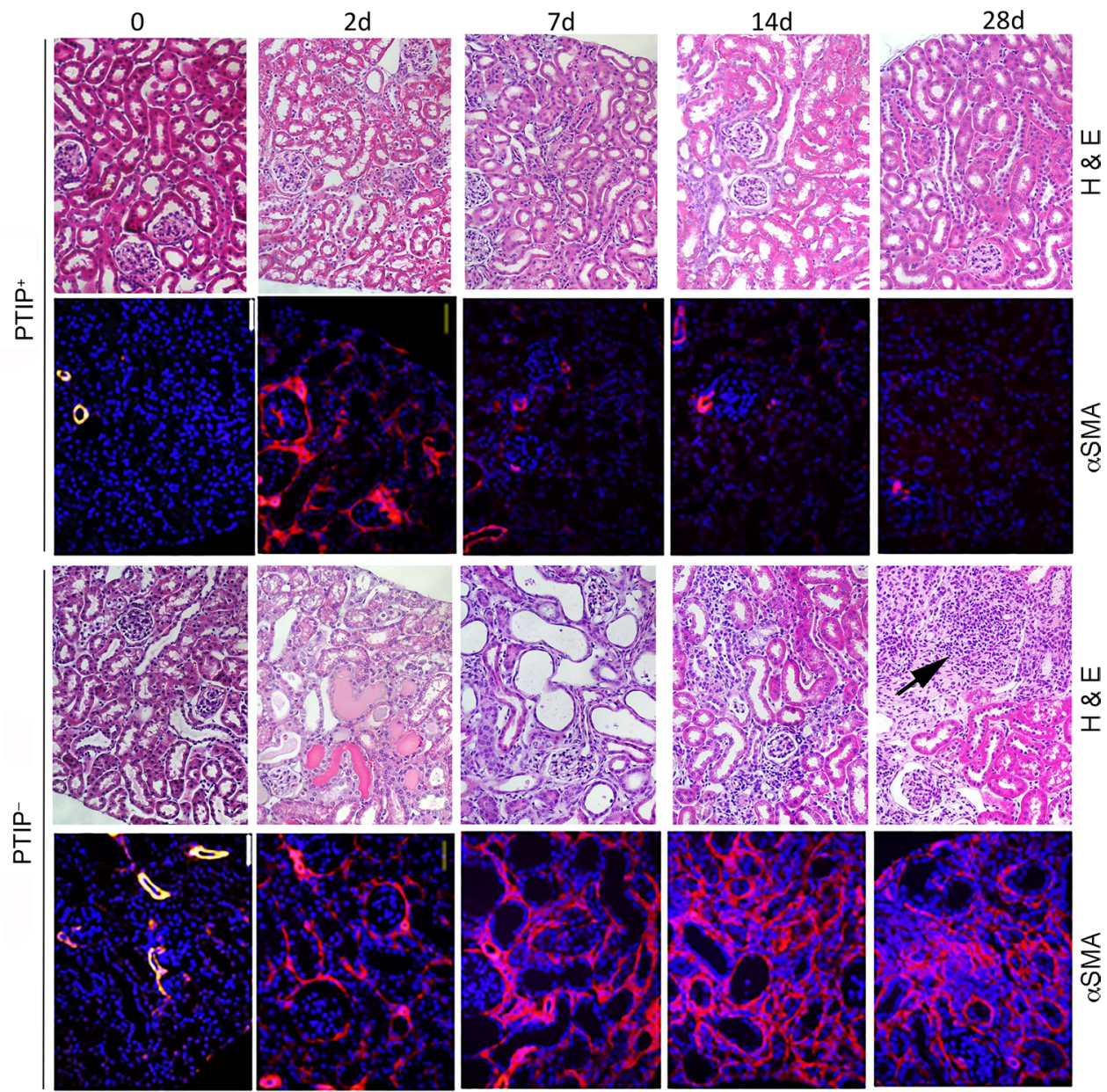

Figure 2. Histology and immunostaining show rapid recovery from AKI in PTIP+ mice but not in PTIP- kidneys. Representative sections for the kidney cortex were stained with H\&E or immunostained for $\alpha$ SMA prior to injury (0) or at 2, 7,14 , and 28 days after AKI. Note expansion of the $\alpha \mathrm{SMA}^{+}$fibroblasts in both genotypes at 2 days but rapid clearing of activated fibroblasts by 7 and 14 days in PTIP+ animals. PTIP- kidneys continued to accumulate $\alpha \mathrm{SMA}^{+}$cells at 7 and 14 days. By 28 days after AKI, PTIP- kidneys exhibited focal areas completely denuded of tubules (arrow).

Additional markers for renal proximal tubules were examined in uninjured kidneys and at various times after AKI (Figure 4). The sodium-dependent phosphate transporter S1c34a3 was expressed primarily in proximal tubules but decreased significantly 2 days after AKI. While $\mathrm{PTIP}^{+}$kidneys exhibited reexpression of Slc34a3 by day 7, PTIP- kidneys did not and still exhibited less Slc34a3 by day 14. Kim1 and Lcn2 expression was strongly induced in both PTIP $^{+}$and PTIP- kidneys at day 2, and it was mostly cleared by day 7 in controls; however, it persisted strongly in PTIP- kidneys through day 14. Aqp1 was widely expressed in the renal cortex but was also reduced in PTIP- kidneys by day 7 in areas denuded of tubules. These data strongly indicate a failure to recover from AKI in $\mathrm{PTIP}^{-}$proximal tubules.

PTIP deletion affects cell proliferation and H3K4 trimethylation. The data suggest that PTIP- proximal tubule cells cannot regenerate efficiently after AKI. In order to examine this more closely, we stained kidneys 2 days after AKI with anti-ki67 to assess proliferation (Figure 5, A-C), when the proliferative response after AKI peaks. The numbers of ki67 ${ }^{+}$cells were reduced 4- to 5-fold in PTIP $^{-}$kidneys compared with $\mathrm{PTIP}^{+}$controls, indicating a clear deficiency in cellular proliferation after injury. Expression of Sox9 is also induced early in regenerating proximal tubule epithelial cells after AKI (25), and it could clearly be seen in PTIP $^{+}$kidneys (Figure 5, D-F). However, no detectable Sox 9 was observed in the PTIP- kidneys. These data indicate that a failure to proliferate and express markers for regeneration underlies the inability to repair tubular damage in the PTIP- $^{-}$kidney.

In cell culture and in developing embryos $(18,26)$, PTIP deletion results in a significant loss of histone $\mathrm{H} 3 \mathrm{~K} 4$ methylation. Thus, we examined levels of $\mathrm{H} 3 \mathrm{~K} 4$ di- and trimethylation by immunostaining and 
A
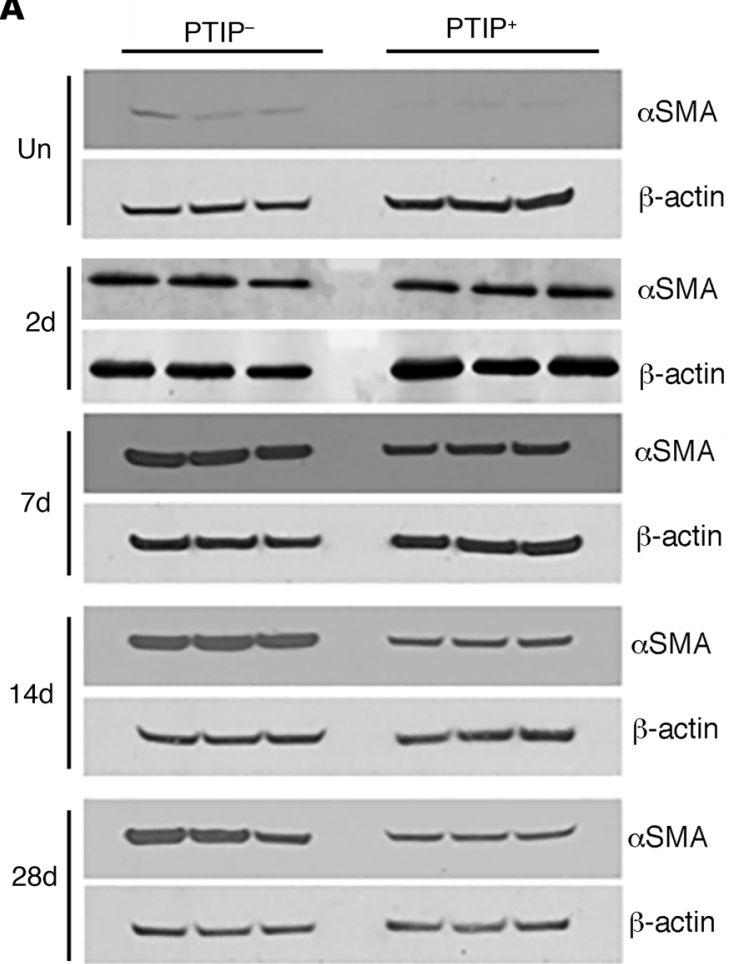
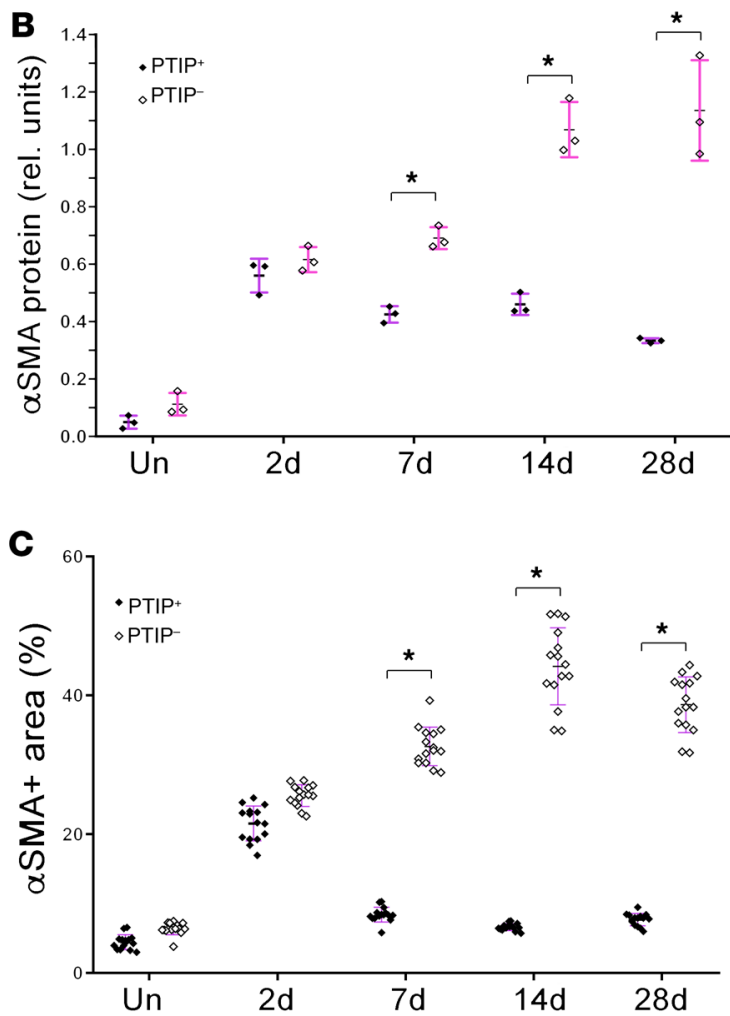

D

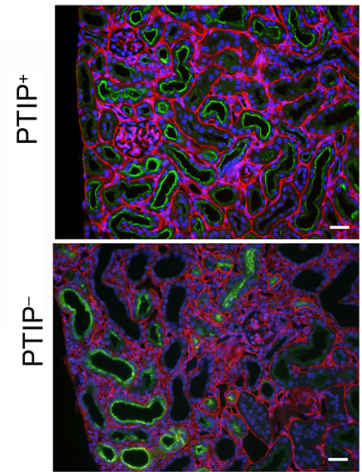

$7 d$

E

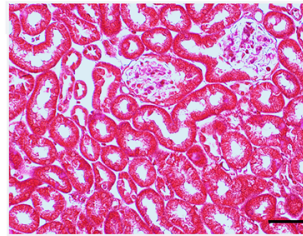

28d, PTIP+

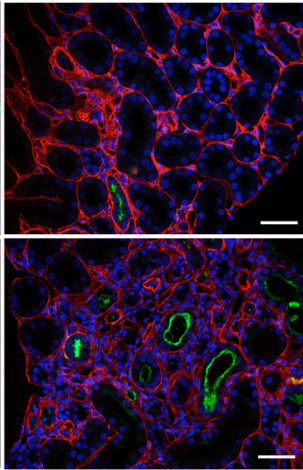

14d

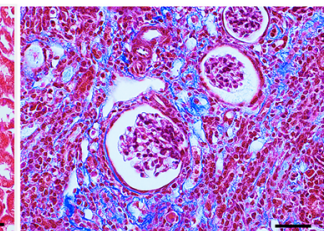

28d, PTIP-

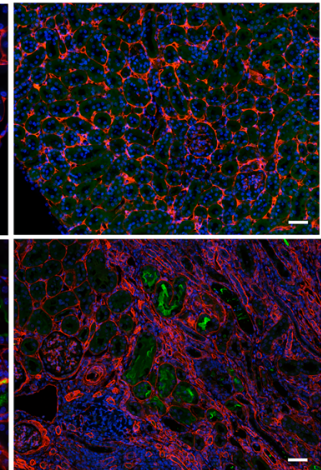

$28 d$

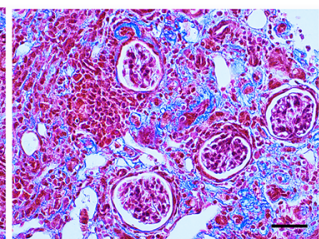

28d, PTIP-

Figure 3. PTIP- kidneys show poor recovery from AKI. (A) Western blots show a rapid increase in $\alpha$ SMA protein levels, relative to loading controls ( $\beta$-actin), at 2 days after AKI in PTIP ${ }^{+}$and PTIP- kidneys. However, $\alpha$ SMA protein levels declined in PTIP+ kidneys over time but remained high in the PTIP- kidneys. (B) Quantitation of the protein levels determined in A. (C) Quantitation of $\alpha$ SMA immunostaining of kidney sections from PTIP+ and PTIP- kidneys at the indicated times after AKI. Day 0 is prior to injury. Data is calculated as percent $\alpha \mathrm{SMA}^{+}$area per microscopic field. Cortical sections from 3 independent animals were counted. Significance was assessed by 2 -way ANOVA using PRISM; ${ }^{*}<0.01$. (D) Immunostaining for Kim1 (green) and ColIV (red) at 7, 14, and 28 days after AKI show strong Kim1 ${ }^{+}$apical surfaces within damaged tubules at 7 days for both PTIP+ and PTIP- kidneys. By 14 days, Kim 1 is barely detectable in PTIP+ kidneys and completely absent by day 28 . PTIP- kidneys still exhibit Kim1 ${ }^{+}$tubules at 14 days and residual Kim1 at 28 days. Also, note that interstitial CollV staining is reduced by 28 days in PTIP+ kidneys. (E) Trichrome staining for cross-linked collagen at 28 days after AKI in PTIP+ and PTIP- kidneys. Representative sections from different mice are shown. 

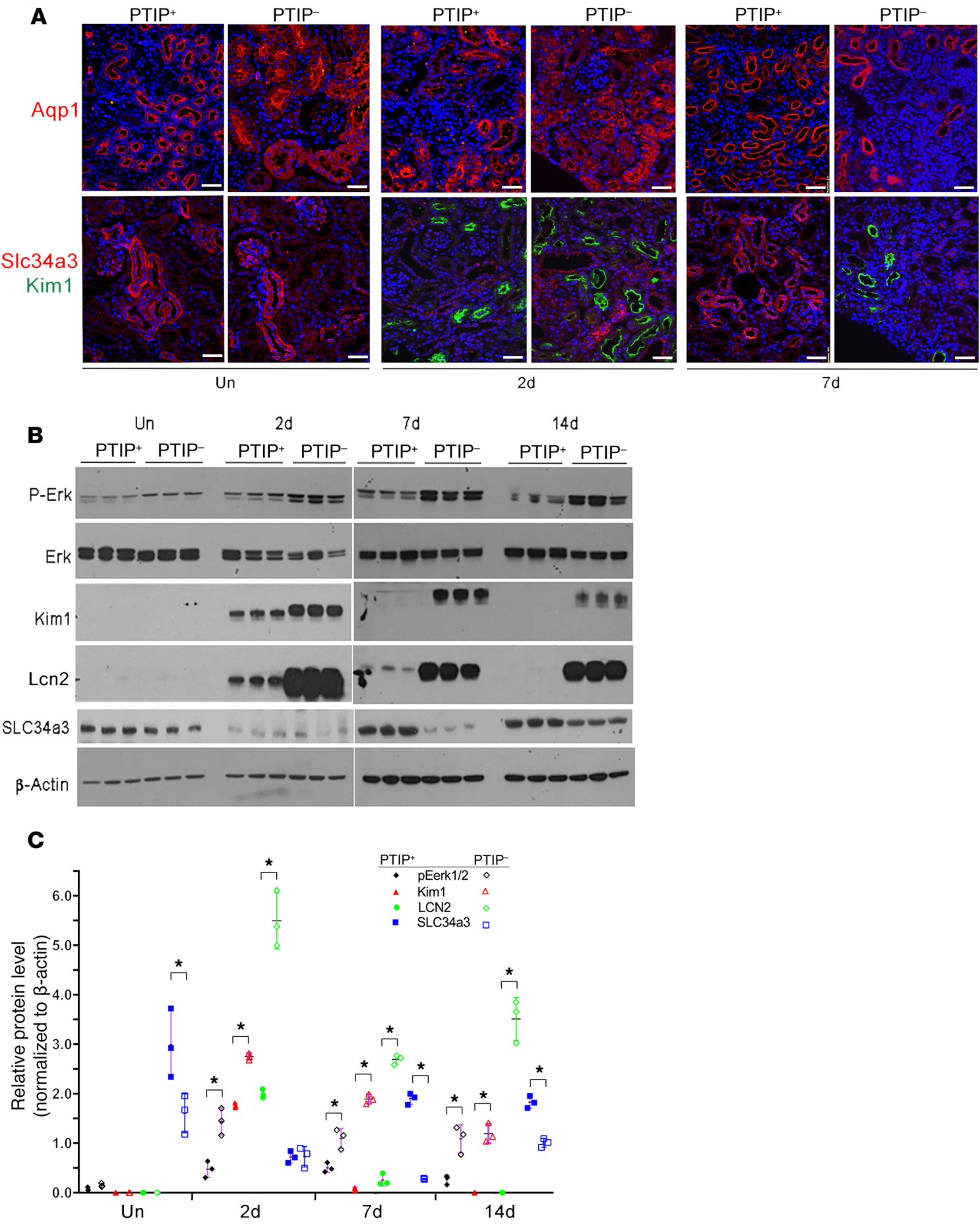

Figure 4. Quantitative analyses of epithelial and injury markers in PTIP- kidneys after AKI. (A) Immunostaining for Aqp1, SLc34a3, and Kim1 in PTIP+ and PTIP- kidneys in uninjured kidneys and 2 and 7 days after AKI. (B) Western blotting of total kidney cortical protein lysates with antibodies against the indicated proteins. Note the persistence of Kim1, P-Erk, and Len2 in PTIP- kidneys and the loss of SIc34a3. (C) Quantitation of Western blots in B by densitometer scanning of different timed exposures, with ${ }^{*} P<0.05$ as determined by 2 -way ANOVA.

Western blotting (Figure 6). Quantitative Western blotting of acid extracted histones showed an approximately $40 \%$ reduction in the global level of $\mathrm{H} 3 \mathrm{~K} 4 \mathrm{me} 2 / 3$ modifications (Figure 6 , A and B), consistent with the known function for PTIP in H3K4 methylation. No significant changes in H3K27 or H4K20 methylation were observed. We also stained kidney sections with antibodies specific for H3K4me3 (Figure 6C) in uninjured and AKI subjected kidneys. While uninjured controls showed a uniform nuclear staining intensity, the PTIP- kidneys had reduced staining intensity — but only in epithelial cells that were also marked 
with EGFP reporter allele, indicating Cre activity and PTIP deletion (Figure 6C). At 2 days after AKI, we noted a reduction in $\mathrm{H} 3 \mathrm{~K} 4 \mathrm{me} 3$ staining in control kidneys, which correlated with Kim1 expression in injured tubules. PTIP- kidneys after AKI continued to show less H3K4me3 staining intensity in nuclei that lacked PTIP. Beyond 28 days after AKI, control kidneys reverted to a more uniform H3K4me3 staining pattern in nuclei, whereas the PTIP- kidneys did not. We also noted that $\mathrm{EGFP}^{+}$cells were seen in PTIPkidneys 28 days after recovery and did not appear to be tubular at all; instead, they appeared to be isolated clusters of cells with no lumens. These data demonstrate reduced levels of histone H3K4 methylation that could impact the ability of cells to reenter mitosis and regenerate damaged proximal tubules.

PTIP mutant cells are located within areas denuded of tubules. Lastly, we utilized a lineage tracing strategy to determine the fate of PTIP ${ }^{-}$cells after AKI. Using the tdTomato-EGFP Cre reporter allele in both $\mathrm{PTIP}^{-}$and $\mathrm{PTIP}^{+}$genetic backgrounds and the Pepck-Cre driver, EGFP ${ }^{+}$cells were found exclusively in the cortex and also stained positive for villin, indicating a proximal tubule phenotype with a brush border (Figure 7A). These mice were examined 28 days after AKI to determine whether $\mathrm{EGFP}^{+}$cells remained. Strikingly, in $\mathrm{PTIP}^{-}$kidneys, many $\mathrm{EGFP}^{+}$cells were found in the interstitium and in regions denuded of tubules. These EGFP ${ }^{+}$cells did not stain for villin or Cadherin-1 (Cdh1), indicating that they had lost their proximal tubule epithelial phenotype (Figure 7, B, C, E, and F). No such cells were found in $\mathrm{PTIP}^{+}$kidneys 28 days after AKI (Figure 7D), as all EGFP ${ }^{+}$cells were found localized within villin ${ }^{+} \mathrm{Cdh} 1^{+}$epithelial tubules. These data suggest that $\mathrm{PTIP}^{-}$cells can lose their epithelial phenotype, perhaps by dedifferentiation, but are unable to proliferate and repopulate the damaged tubules. Instead, some isolated PTIP $^{-}$cells that survive have lost expression of villin and Cdh1, and they appear as single cells or cluster with no obvious epithelial polarity. While these data suggest an epithelial-to-mesenchymal transition, the $\mathrm{EGFP}^{+}$interstitial cells found in PTIP $^{-}$kidneys do not express $\alpha$ SMA or PDGFR $\alpha$ (data not shown), typical markers for interstitial fibroblasts during injury. Instead, the $\mathrm{EGFP}^{+}$cells that have lost the epithelial phenotype may reflect damaged, dedifferentiated cells that cannot proliferate and that cannot revert to the fully differentiated phenotype.

\section{Discussion}

The epigenetic writers M113 and M114 (also known as KMT2C and KMT2D) are histone H3K4 methyltransferases that mark enhancers during development and cell lineage specification (27). These Mlls are homologues of the Drosophila Trithorax-related (Trr) methyltransferase and function within a large, conserved, multiprotein complex $(28,29)$. Among the cofactors necessary for Mll-mediated H3K4 methylation are Ash2, Wdr5, Rppb5 (30), and PTIP $(17,18)$. The multi-BRCT domain-containing protein PTIP, encoded by the Paxip1 gene, is thought to bind phospho-serine residues $(31,32)$ and link M1l3/4 to DNA binding proteins to imprint H3K4 mono-, di-, and trimethylation in mammals (18) and in Drosophila (26). The importance of Mll-mediated H3K4 methylation in development is clearly demonstrated by early postgastrulation lethality in PTIP-null mutant mice (33). In leukemia, PTIP is needed to set a HoxA9-dependent pattern of histone methylation at enhancer sequences (34). However, PTIP deletion in terminally differentiated cells does not lead to catastrophic consequences in mice, as observed in cardiomyocytes (21) or glomerular podocytes (19). In mature $\operatorname{IgM}^{+}$B cells, deletion of PTIP impairs de novo H3K4 methylation at transcriptional start sites for new heavy chain constant domains upon isotype switching and prevents chromosomal looping to the $\operatorname{IgM}$ enhancer $(35,36)$. In the developing kidney, temporal deletion of PTIP clearly impacts de novo gene expression without grossly affecting tissue-specific markers expressed prior to deletion, clearly demonstrating an epigenetic effect on cellular memory (37). However, none of these model systems addressed whether a regenerating cell after injury requires a resetting of histone methylation marks for recovery and repair. Given that regenerating cells are derived from fully differentiated epithelial cells, whose epigenetic marks are presumably imprinted and stable, the need for PTIP activity suggests an epigenetic resetting or de novo methylation of specific histone marks during regeneration.

The adult kidney nephron consists of differentiated, specialized epithelial cells, from the podocytes and parietal epithelium of Bowman's capsule, to proximal and distal tubules, to the collecting ducts. In response to AKI caused by nephrotoxins or ischemia, only the proximal tubules are able to regenerate. Lineage tracing analysis clearly demonstrated that, in response to sublethal doses of AKI, surviving proximal tubule cells were able to reexpress developmental control genes, such as Sox9 $(25,38)$ and Pax2 (39); enter mitosis; and repopulate the tubular basement membrane along sites of injury $(11,40)$. If enhancer sequences are marked by specific histone methylation patterns in differentiated cells and such cells are 

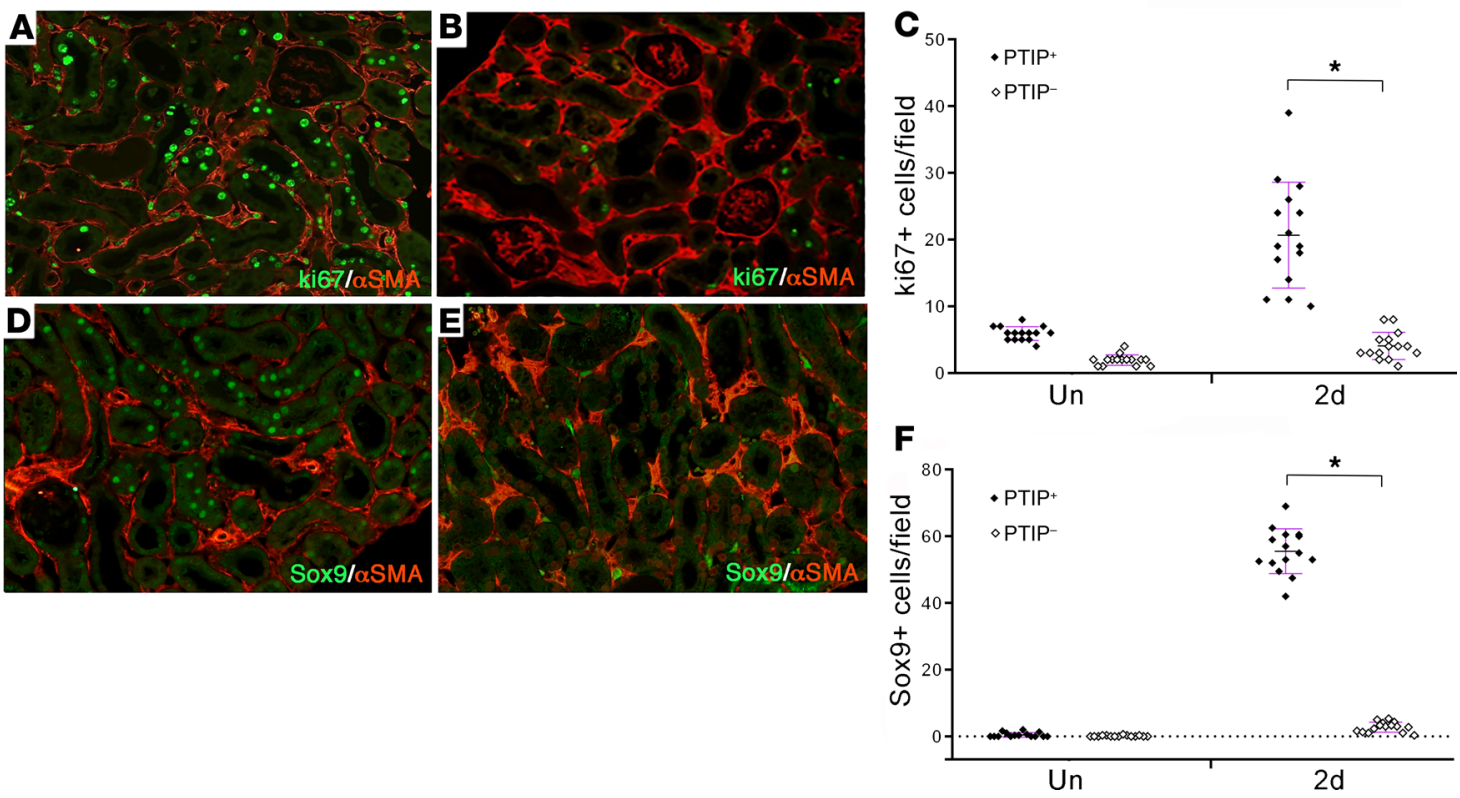

Figure 5. Inhibition of Sox9 activation and cell proliferation in PTIP- proximal tubules after AKI. (A and B) Cell proliferation was assayed by ki67 immunostaining (green) at 2 days after AKI in PTIP+ (A) and PTIP- (B) kidneys, both costained for aSMA (red). (C) Quantitation of data collected from 3 independent animals and expressed as number of ki67+ cells per low-powered field. (D and E) Immunostaining for Sox9 (green) and $\alpha$ SMA (red) in PTIP+ kidneys (D), or in or PTIP- (E) kidney sections taken 2 days after AKI. (F) Quantitation of 5 sections per animal from 3 independent mice. Note significant numbers of Sox9+ cells in PTIP+ kidneys at 2 days, indicating regeneration, whereas PTIP- kidneys had few, if any, Sox9 ${ }^{+}$cells 2 days after AKI. ${ }^{*} P<0.001$ by 2 -way ANOVA.

then pushed to reenter mitosis, the question remains whether such patterns are replicated in the absence of PTIP-mediated M113/4 activity. Failure to replicate such patterns in regenerating cells would indicate a loss of epigenetic memory and a potential alteration of phenotypes. This is precisely what we observed in PTIP- proximal tubule cells after FA-induced AKI. Very few cells were in mitosis and none expressed the developmental marker Sox 9 at 2 days after injury. The end result was scarring and loss of nephrons in animals that survived the initial dose of the nephrotoxin. It has been proposed that repeated bouts of AKI can lead to chronic renal insufficiency $(41,42)$. While we have not tested this in our PTIP- model directly, the increased mortality observed with just a single dose and the fibrosis seen in surviving animals strongly suggest that AKI can promote pathology associated with chronic renal interstitial disease.

Our data strongly suggest that defects in histone methylation impair the ability to regenerate proximal tubules. More specifically, it is H3K4 methylation that appears most affected in PTIP mutants. Global levels of H3K4me2/3 appeared reduced, although we cannot distinguish whether this was due to a complete absence at a subset of promoters and enhancers or whether this was just a proportionate reduction at all $\mathrm{H} 3 \mathrm{~K} 4 \mathrm{me}$ sites. PTIP mutant proximal tubules already show low levels of H3K4me3 that remain low after $\mathrm{AKI}$ and recovery, which - in the PTIP- kidneys - is characterized mostly by scarring and formation of fibrotic plaques. While immunostaining for $\mathrm{H} 3 \mathrm{~K} 4 \mathrm{me} 3 \mathrm{can}$ be difficult to quantitate from section to section, using the EGFP Cre reporter alleles allowed us to examine both $\mathrm{PTIP}^{-}$cells and $\mathrm{PTIP}^{+}$cells in the same kidney sections taken from the PTIP- $^{-}$animals for a direct comparison.

Epigenetic changes have been described in models of AKI $(43,44)$, but it was not clear whether these were necessary for repair or what proteins may be driving such changes. Our data suggest that H3K4me 3 can be suppressed in differentiated proximal tubules without gross abnormalities evident in renal histology or function, as observed in uninjured PTIP- kidneys. The data also suggest that the Pepck-Cre-mediated PTIP deletion may be occurring so late in differentiation that sufficient $\mathrm{H} 3 \mathrm{~K} 4 \mathrm{me}$ has already occurred and is stable enough for maintaining renal function. However, expression of genes induced by AKI is inhibited in these injured tubules, suggesting that de novo $\mathrm{H} 3 \mathrm{~K} 4 \mathrm{me}$, as mediated by the PTIP-MLL3/4 complex, is essential for driving cells back into mitosis and activation genes such as Sox9, which are essential for regeneration.

Other examples of epigenetic memory in differentiated renal epithelial cells include the lack of phenotype upon deletion of the $P k d 1$ gene in adults. Deletion of either $P k d 1$ (45) or $P k d 2$ (46) in developing 


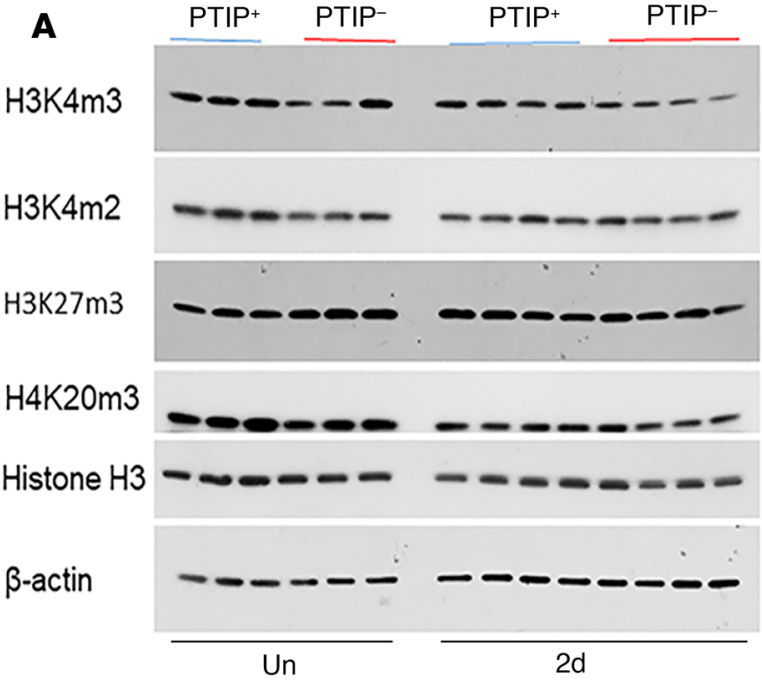

B
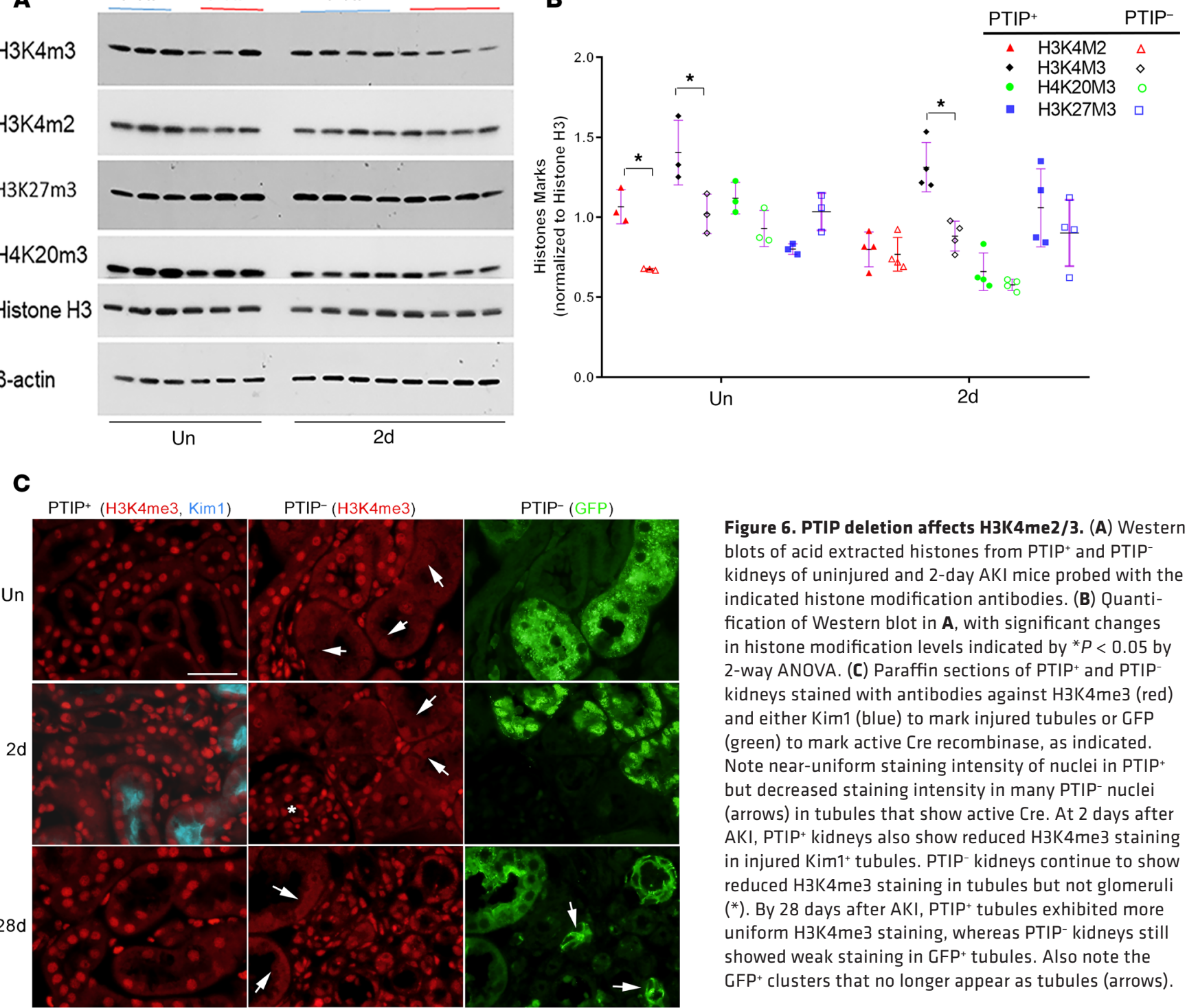

Figure 6. PTIP deletion affects H3K4me2/3. (A) Western blots of acid extracted histones from $\mathrm{PTIP}^{+}$and PTIPkidneys of uninjured and 2-day AKI mice probed with the indicated histone modification antibodies. (B) Quantification of Western blot in $\mathbf{A}$, with significant changes in histone modification levels indicated by ${ }^{*} P<0.05$ by 2-way ANOVA. (C) Paraffin sections of PTIP+ and PTIPkidneys stained with antibodies against $\mathrm{H} 3 \mathrm{~K} 4 \mathrm{me} 3$ (red) and either Kim1 (blue) to mark injured tubules or GFP (green) to mark active Cre recombinase, as indicated. Note near-uniform staining intensity of nuclei in $\mathrm{PTIP}^{+}$ but decreased staining intensity in many PTIP- nuclei (arrows) in tubules that show active Cre. At 2 days after AKI, PTIP+ kidneys also show reduced $\mathrm{H} 3 \mathrm{~K} 4 \mathrm{me} 3$ staining in injured $\mathrm{Kim}^{+}$tubules. PTIP- kidneys continue to show reduced $\mathrm{H} 3 \mathrm{~K} 4 \mathrm{me} 3$ staining in tubules but not glomeruli $\left({ }^{*}\right)$. By 28 days after AKI, PTIP+ tubules exhibited more uniform H3K4me3 staining, whereas PTIP- kidneys still showed weak staining in $\mathrm{GFP}^{+}$tubules. Also note the GFP' clusters that no longer appear as tubules (arrows).

kidneys promotes rapid and widespread renal cyst formation. While it was initially proposed that renal cysts form as a result of a loss of heterozygosity at the $P k d 1$ or $P k d 2$ locus $(47,48)$, induced conditional null mutations in adult mice do not form cysts very efficiently (13). However, upon renal AKI, mice whose renal epithelial cells are $P k d 1$-null or are missing primary cilia entirely generate cysts in the recovery phase after injury $(14,15,49,50)$. These results suggest that reentry into mitosis is a prerequisite for cystogenesis in cells that have lost both copies of either $P k d 1$ or $P k d 2$. Thus, development of the cystic disease phenotype requires not only a loss of heterozygosity in either polycystic kidney gene, but also an acute injury event that drives some mutant cells into the cell cycle. Similarly, regulation of $P k d 2$ by the transcription factor HNF1 $\beta$ is seen in developing kidneys, but deletion of HNF1 $\beta$ in terminally differentiated cells has no affect on $\mathrm{Pkd} 2$ gene expression (16). Once again, when kidneys are injured, HNF1 $\beta$ is required to activate or maintain expression of $\mathrm{Pkd} 2$ after recovery. These data and ours indicate that the H3K4 methylation machinery, while not immediately required for terminally differentiated cells, remains essential if and when such cells are required to divide again. This can have important clinical implications as more efforts are geared toward developing anticancer drugs that target epigenetic modifying complexes, such as the Mlls in leukemia or Ezh2 in prostate and other cancers $(51,52)$.

While the loss of PTIP in proximal tubules did not lead to a reduction in measurable renal functions, we did observe an increase in mortality after FA, suggesting that the $\mathrm{PTIP}^{-}$animals were partially sensitized to AKI. The detection of Lcn2/Ngal in PTIP- kidneys prior to injury also was consistent with sensitization, as 


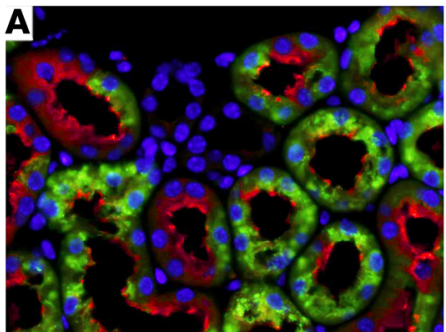

un., PTIP- (GFP/villin)

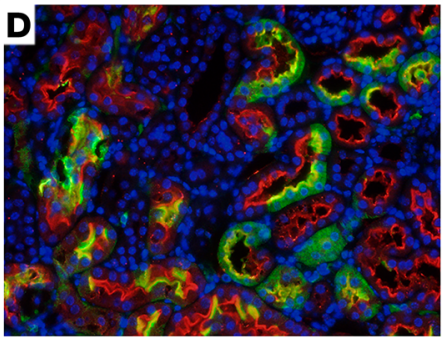

28d AKI, PTIP+(GFP/villin)

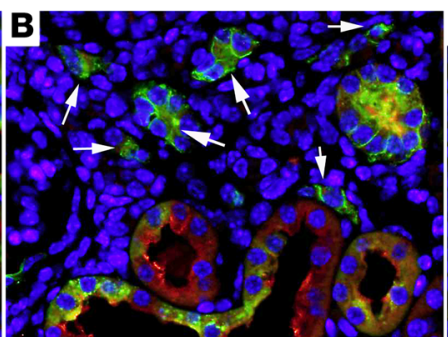

28d AKI, PTIP- (GFP/villin)

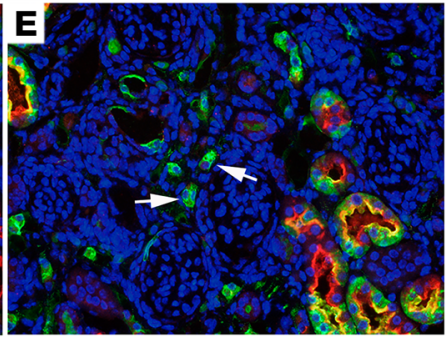

28d AKI, PTIP- (GFP/villin)

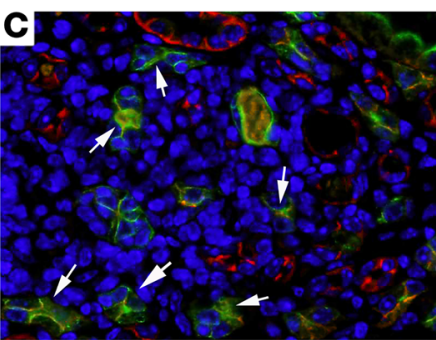

28d AKI, PTIP- (GFPICdh1)

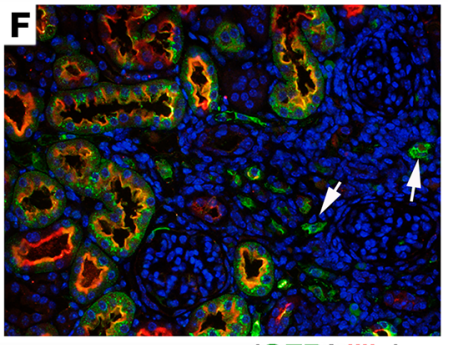

28d AKI, PTIP-(GFP/villin)

Figure 7. Lineage tracing of proximal tubule cells before and after AKI. (A) Prior to AKI, EGFP marks all $\mathrm{Cre}^{+}$ proximal tubule cells in PTIP- kidneys that are also positive for the brush border protein villin (red). No EGFP+ ${ }^{+}$cells are seen in the interstitium. (B and C) EGFP lineage marked cells 28 days after AKI in PTIP- kidneys costained for villin (B, red) or Cadherin-1 (C, red). Note EGFP+ ${ }^{+}$cells (arrows) within the interstitium or in regions denuded of tubules. Such surviving PTIP- cells have lost their epithelial makers. (D) Lineage trace in PTIP+ kidneys 28 days after AKI indicate that all EGFP+ cells are villin+ and located within tubules. (E and F) Sections from 2 independent PTIP kidneys 28 days after AKI exhibit multiple EGFP+ cells (arrows) located outside of epithelial tubules and negative for villin (red) located within the interstitium.

Lcn2 is considered one of the earliest biomarkers for injury (53). The fact that we saw low levels of expression in the more distal nephron is consistent with the known localization of the highest levels of Lcn2 after AKI, which are more distal despite the injury being more proximal (54). The reduction in proliferating cells at early times after injury and the lack of Sox9 induction must underlie the failure of surviving proximal tubule cells to repair. Still, many PTIP- cells survive the initial insult but end up in the interstitium, having lost their epithelial makers. Such PTIP- cells did not reflect an epithelial-to-mesenchymal transition, as no expression of $\alpha$ SMA or PDGFR $\alpha$ costained with the lineage tracing marker EGFP. Our data suggest that PTIP- and MLL-mediated H3K4 methylation must be needed for the activation of regeneration-specific genes and pathways. Thus, patterns of histone methylation appear sufficiently stable in nondividing cells to maintain phenotypes, but a response to injury and reentry into mitosis must require new H3K4 methylation.

\section{Methods}

Animals. Mice were housed in a specific pathogen-free facility with a 12-hour light, 12-hour dark cycle and given free access to food and water. PTIP- were obtained by crossing the Paxip ${ }^{f l / l}$ mice strain (55) with the report-

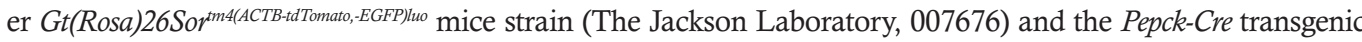
mice. The Pepck-Cre transgenic mice were generated by targeted single-copy transgenesis in embryonic stem cells (a gift from Volker H. Haase, Vanderbilt University, Nashville, Tennessee, USA) (22). For genotyping, genomic DNA was prepared by a standard method and amplified by PCR using the following primer pair sets: EGFP, forward, 5' - GGGCGATGCCACCTACGGCAAGCTGACCCT - 3', and reverse, 5' - CCGTCCTCCTTGAAGTCGATGCCCTTCAGC - 3'; PTIP, forward, 5' - GGTTCTCTTGCAGCATCTCC - 3'; PTIP f/fl $^{\text {, }}$ reverse, 5' - GGGAACTGATCTTCGATGAGG - 3'; PTIP'-, reverse, 5' - CTACCGTACAATCCACCCTAAG - 3'; Cre, forward, 5' - CGAGTGATGAGGTTCGCAAG - 3', and reverse, 5' - TGAGTGAACGAACCTGGTCG - 3'; tdTomato WT, forward, 5' - CTCTGCTGCCTCCTGGCTTCT - 3'; WT, reverse, 5' - CGAGGCGGATCACAAGCAATA - 3'; and floxed, reverse, 5' - TCAATGGGC GGGGGTCGTT - 3'.

For the AKI model, PTIP $^{-}$mice and control $\mathrm{PTIP}^{+}$mice were age matched and injected i.p. with a single dose of $250 \mathrm{mg} / \mathrm{Kg}$ of $\mathrm{FA}$ in total volume of $500 \mu \mathrm{L}$ of $0.15 \mathrm{M} \mathrm{NaHCO}_{3}$ as described previously (56). Because of the high percentage of death, $\mathrm{PTIP}^{-}$mice were put under constant observation for the 
first 48 hours after injection. Mice were then sacrificed at 2, 7, 14, or 28 days after injury, and blood samples were obtained from the abdominal aorta for renal function tests. Immediately, mice were perfused with cold PBS to improve fixation and tissue processing. Three to 4 age-matched mice were taken of each genotype, and kidneys were harvested; 1 kidney of each was used for histology, and the other kidney was freshly frozen and used for protein and RNA analysis.

Histology and immunostaining. Kidney slides were fixed overnight in 4\% PFA and either processed for OCT or paraffin-embedded, and sections of $5 \mu \mathrm{m}$ were used for histology (H\&E, Masson's trichrome). Immunofluorescence detection was done using a modified version of published protocols (57). Paraffin sections of $5 \mu \mathrm{m}$ were rehydrated and stained with mouse, monoclonal anti- $\alpha \mathrm{SMA}-\mathrm{Cy} 3$ conjugated (MilliporeSigma, C6198), rabbit anti-Collagen IV (Chemicon, AB756P), goat anti-Kim1 (R\&D Systems, anti-mTIM1, AF1817), mouse anti-GFP (Santa Cruz Biotechnology Inc., sc-9996), goat anti-villin (Santa Cruz Biotechnology Inc., sc-7672), mouse anti-E-Cadherin (BD Biosciences, 610182), goat anti-Sox9 (R\&D Systems, AF3075), rat anti-ki67 (BioLegend, 562402), and goat anti-mouse lipocalin-2/NGAL (R\&D Systems AF1857). For histone methylation staining, we utilized rabbit anti-H3K4me3 (Cell Signaling Technology, 9751) and rabbit anti-H3K4me2 (Abcam, ab7766). Rabbit anti-PTIP antibodies were generated against the GST-fused mouse PTIP residues 316-591 and purified on protein A agarose (58). Images were collected using an Olympus DP72 camera (Olympus America Inc.). Saved images were analyzed using MetaMorph Version 7.7.4 (Molecular Devices Inc.) and confirmed with ImageJ (NIH) analysis. The sampling area was outlined, and MetaMorph calculated the proportion of surface areas with signals above a given threshold. At least 15 images were used to calculate the average surface area for each time point and repeated at least once having the same results.

Western blot. Half of each fresh-frozen kidney was used for protein analysis, and kidney extracts were prepared as briefly described in refs. 59 and 60. Tissue was homogenized in RIPA buffer (R0278, MilliporeSigma) and supplemented with freshly added protease inhibitor cocktails (Roche Diagnostics). Crude lysates were centrifuged at $14,000 \mathrm{~g}$ for 10 minutes at $4^{\circ} \mathrm{C}$, and the protein concentration was measured using the BCA Protein Assay Kit (23227, Pierce). Samples were mixed in 2× SDS/PAGE sample buffer, separated on gradient precast polyacrylamide gels, and transferred to PVDF membranes (MilliporeSigma, IPFL00010). For acid extraction of histones, we used the Abcam kit (ab113476) according the manufacturer's instructions. Specific proteins were detected with primary antibodies and horseradish peroxidaseconjugated secondary antibodies (GE Healthcare) using Western Lightning Enhanced Chemiluminescence (Pierce). The following antibodies were purchased: mouse anti- $\alpha$ SMA (MilliporeSigma, A5228), rabbit anti- $\beta$-actin (Sigma-Aldrich, A2066), rabbit anti-GAPH (Cell Signaling Technology, 2118S), rabbit antiH3K4me3 (Abcam, ab8580), rabbit anti-Slc34a3 (Thermo Fisher Scientific, PA5-95706), and rabbit antiPTIP (61). Blots were scanned and analyzed for band intensities by using ImageJ software analysis.

Renal function tests. In vitro quantification of BUN and creatinine in serum was performed with LIASYS 330 Chemistry Analyzer, and we used standard proved protocols for mouse serum samples. Services were provided by the In-Vivo Animal Core (IVAC) Animal Diagnostic Laboratory, University of Michigan. Specific product parameters and procedures are found in the LIASYS 330 instrument software, showing a BUN normal range of $5.15-30.70 \mathrm{mg} / \mathrm{dL}$ and a creatinine normal range of $0.09-0.40 \mathrm{mg} / \mathrm{dL}$.

Statistics. Each experiment was performed using at least 3 mice per group and repeated in entirety at least once, using different software measurement and producing similar results. For micrographs, specific areas were measured over 15-20 images. For cell proliferation counts, nuclei were counted from 5 low-power fields for 3 different kidneys. For Western blotting, 3 independent kidney lysates were run, and protein levels were measured by densitometry scanning of autoradiographs at appropriate exposures in the linear range. Averages and SDs were calculated using GraphPad Prism7 version $7.0 \mathrm{~d}$, and significance was assessed by 2 -way ANOVA using the PRISM software. $P<0.05$ was considered to be significant.

Study approval. All animals used complied with the Guide for the Care and Use of Laboratory Animals (National Academies Press, 2011) and approved by the University Committee on Use and Care of Animals at the University of Michigan.

\section{Author contributions}

AS and GRD designed the experiment. APK, MA, and AS collected data and analyzed images. AS and AML bred and maintained the mice. GRD and AML drafted and edited the manuscript. 


\section{Acknowledgments}

We thank V. Haase for the Pepck-Cre mice and S. Patel for helpful discussion. This work was supported by NIH grants DK054740 and DK073722 to GRD.

Address correspondence to: Gregory R. Dressler, 109 Zina Pitcher Drive, BSRB 2049, Ann Arbor, Michigan 48109, USA. Phone: 734.764.6490; Email: dressler@umich.edu.

1. Perino M, Veenstra GJ. Chromatin Control of Developmental Dynamics and Plasticity. Dev Cell. 2016;38(6):610-620.

2. Soshnev AA, Josefowicz SZ, Allis CD. Greater Than the Sum of Parts: Complexity of the Dynamic Epigenome. Mol Cell. 2016;62(5):681-694.

3. Bernstein BE, et al. A bivalent chromatin structure marks key developmental genes in embryonic stem cells. Cell. 2006;125(2):315-326.

4. Cantone I, Fisher AG. Epigenetic programming and reprogramming during development. Nat Struct Mol Biol. 2013;20(3):282-289.

5. Lanzuolo C, Orlando V. Memories from the polycomb group proteins. Annu Rev Genet. 2012;46:561-589.

6. Ringrose L, Paro R. Polycomb/Trithorax response elements and epigenetic memory of cell identity. Development. 2007;134(2):223-232.

7. Schuettengruber B, Martinez AM, Iovino N, Cavalli G. Trithorax group proteins: switching genes on and keeping them active. Nat Rev Mol Cell Biol. 2011;12(12):799-814.

8. Zuk A, Bonventre JV. Acute Kidney Injury. Annu Rev Med. 2016;67:293-307.

9. Chang-Panesso M, Humphreys BD. Cellular plasticity in kidney injury and repair. Nat Rev Nephrol. 2017;13(1):39-46.

10. Duffield JS, Humphreys BD. Origin of new cells in the adult kidney: results from genetic labeling techniques. Kidney Int. 2011;79(5):494-501.

11. Humphreys BD, Czerniak S, DiRocco DP, Hasnain W, Cheema R, Bonventre JV. Repair of injured proximal tubule does not involve specialized progenitors. Proc Natl Acad Sci USA. 2011;108(22):9226-9231.

12. Kusaba T, Lalli M, Kramann R, Kobayashi A, Humphreys BD. Differentiated kidney epithelial cells repair injured proximal tubule. Proc Natl Acad Sci USA. 2014;111(4):1527-1532.

13. Piontek K, Menezes LF, Garcia-Gonzalez MA, Huso DL, Germino GG. A critical developmental switch defines the kinetics of kidney cyst formation after loss of Pkd1. Nat Med. 2007;13(12):1490-1495.

14. Takakura A, et al. Renal injury is a third hit promoting rapid development of adult polycystic kidney disease. Hum Mol Genet. 2009;18(14):2523-2531.

15. Patel V, et al. Acute kidney injury and aberrant planar cell polarity induce cyst formation in mice lacking renal cilia. Hum Mol Genet. 2008;17(11):1578-1590.

16. Verdeguer F, et al. A mitotic transcriptional switch in polycystic kidney disease. Nat Med. 2010;16(1):106-110.

17. Cho YW, et al. PTIP associates with MLL3- and MLL4-containing histone H3 lysine 4 methyltransferase complex. J Biol Chem. 2007;282(28):20395-20406.

18. Patel SR, Kim D, Levitan I, Dressler GR. The BRCT-domain containing protein PTIP links PAX2 to a histone H3, lysine 4 methyltransferase complex. Dev Cell. 2007;13(4):580-592.

19. Lefevre GM, Patel SR, Kim D, Tessarollo L, Dressler GR. Altering a histone H3K4 methylation pathway in glomerular podocytes promotes a chronic disease phenotype. PLoS Genet. 2010;6(10):e1001142.

20. Stein AB, et al. The PTIP-Associated Histone Methyltransferase Complex Prevents Stress-Induced Maladaptive Cardiac Remodeling. PLoS ONE. 2015;10(5):e0127839.

21. Stein $\mathrm{AB}$, et al. Loss of $\mathrm{H} 3 \mathrm{~K} 4$ methylation destabilizes gene expression patterns and physiological functions in adult murine cardiomyocytes. J Clin Invest. 2011;121(7):2641-2650.

22. Rankin EB, Tomaszewski JE, Haase VH. Renal cyst development in mice with conditional inactivation of the von Hippel-Lindau tumor suppressor. Cancer Res. 2006;66(5):2576-2583.

23. Mishra J, et al. Neutrophil gelatinase-associated lipocalin (NGAL) as a biomarker for acute renal injury after cardiac surgery. Lancet. 2005;365(9466):1231-1238

24. Mishra J, et al. Identification of neutrophil gelatinase-associated lipocalin as a novel early urinary biomarker for ischemic renal injury. J Am Soc Nephrol. 2003;14(10):2534-2543.

25. Kang HM, Huang S, Reidy K, Han SH, Chinga F, Susztak K. Sox9-Positive Progenitor Cells Play a Key Role in Renal Tubule Epithelial Regeneration in Mice. Cell Rep. 2016;14(4):861-871.

26. Fang M, Ren H, Liu J, Cadigan KM, Patel SR, Dressler GR. Drosophila ptip is essential for anterior/posterior patterning in development and interacts with the PcG and trxG pathways. Development. 2009;136(11):1929-1938.

27. Sze CC, Shilatifard A. MLL3/MLL4/COMPASS Family on Epigenetic Regulation of Enhancer Function and Cancer. Cold Spring Harb Perspect Med. 2016;6(11):a026427.

28. Shilatifard A. Molecular implementation and physiological roles for histone H3 lysine 4 (H3K4) methylation. Curr Opin Cell Biol. 2008;20(3):341-348.

29. Froimchuk E, Jang Y, Ge K. Histone H3 lysine 4 methyltransferase KMT2D. Gene. 2017;627:337-342.

30. Dou Y, et al. Regulation of MLL1 H3K4 methyltransferase activity by its core components. Nat Struct Mol Biol. 2006;13(8):713-719.

31. Manke IA, Lowery DM, Nguyen A, Yaffe MB. BRCT repeats as phosphopeptide-binding modules involved in protein targeting. Science. 2003;302(5645):636-639.

32. Yu X, Chini CC, He M, Mer G, Chen J. The BRCT domain is a phospho-protein binding domain. Science. 2003;302(5645):639-642.

33. Cho EA, Prindle MJ, Dressler GR. BRCT domain-containing protein PTIP is essential for progression through mitosis. Mol Cell Biol. 2003;23(5):1666-1673. 
34. Sun Y, et al. HOXA9 Reprograms the Enhancer Landscape to Promote Leukemogenesis. Cancer Cell. 2018;34(4):643-658.e5.

35. Daniel JA, et al. PTIP promotes chromatin changes critical for immunoglobulin class switch recombination. Science. 2010;329(5994):917-923.

36. Schwab KR, Patel SR, Dressler GR. Role of PTIP in class switch recombination and long-range chromatin interactions at the immunoglobulin heavy chain locus. Mol Cell Biol. 2011;31(7):1503-1511.

37. Ranghini EJ, Dressler GR. Evidence for intermediate mesoderm and kidney progenitor cell specification by Pax2 and PTIP dependent mechanisms. Dev Biol. 2015;399(2):296-305.

38. Kumar S, et al. Sox9 Activation Highlights a Cellular Pathway of Renal Repair in the Acutely Injured Mammalian Kidney. Cell Rep. 2015;12(8):1325-1338.

39. Imgrund $\mathrm{M}$, et al. Re-expression of the developmental gene Pax-2 during experimental acute tubular necrosis in mice 1 . Kidney Int. 1999;56(4):1423-1431

40. Humphreys BD, et al. Intrinsic epithelial cells repair the kidney after injury. Cell Stem Cell. 2008;2(3):284-291.

41. Bonventre JV, Yang L. Cellular pathophysiology of ischemic acute kidney injury. J Clin Invest. 2011;121(11):4210-4221.

42. Yang L, Humphreys BD, Bonventre JV. Pathophysiology of acute kidney injury to chronic kidney disease: maladaptive repair. Contrib Nephrol. 2011;174:149-155.

43. Guo C, Dong G, Liang X, Dong Z. Epigenetic regulation in AKI and kidney repair: mechanisms and therapeutic implications. Nat Rev Nephrol. 2019;15(4):220-239.

44. Sharifian R, et al. Distinct patterns of transcriptional and epigenetic alterations characterize acute and chronic kidney injury. Sci Rep. 2018;8(1):17870.

45. Lu W, et al. Perinatal lethality with kidney and pancreas defects in mice with a targetted Pkd1 mutation. Nat Genet. 1997;17(2):179-181.

46. Wu G, et al. Somatic inactivation of Pkd2 results in polycystic kidney disease. Cell. 1998;93(2):177-188.

47. Qian F, Watnick TJ, Onuchic LF, Germino GG. The molecular basis of focal cyst formation in human autosomal dominant polycystic kidney disease type I. Cell. 1996;87(6):979-987.

48. Pei Y, et al. Somatic PKD2 mutations in individual kidney and liver cysts support a "two-hit" model of cystogenesis in type 2 autosomal dominant polycystic kidney disease. J Am Soc Nephrol. 1999;10(7):1524-1529.

49. Bastos AP, et al. Pkd1 haploinsufficiency increases renal damage and induces microcyst formation following ischemia/reperfusion. J Am Soc Nephrol. 2009;20(11):2389-2402.

50. Happé $\mathrm{H}$, et al. Toxic tubular injury in kidneys from Pkd1-deletion mice accelerates cystogenesis accompanied by dysregulated planar cell polarity and canonical Wnt signaling pathways. Hum Mol Genet. 2009;18(14):2532-2542.

51. Tsai CT, So CW. Epigenetic therapies by targeting aberrant histone methylome in AML: molecular mechanisms, current preclinical and clinical development. Oncogene. 2017;36(13):1753-1759.

52. Yamagishi M, Uchimaru K. Targeting EZH2 in cancer therapy. Curr Opin Oncol. 2017;29(5):375-381.

53. Devarajan P. Biomarkers for the early detection of acute kidney injury. Curr Opin Pediatr. 2011;23(2):194-200.

54. Paragas N, et al. The Ngal reporter mouse detects the response of the kidney to injury in real time. Nat Med. 2011;17(2):216-222.

55. Kim D, Wang M, Cai Q, Brooks H, Dressler GR. Pax transactivation-domain interacting protein is required for urine concentration and osmotolerance in collecting duct epithelia. J Am Soc Nephrol. 2007;18(5):1458-1465.

56. Higashi AY, Aronow BJ, Dressler GR. Expression Profiling of Fibroblasts in Chronic and Acute Disease Models Reveals Novel Pathways in Kidney Fibrosis. J Am Soc Nephrol. 2019;30(1):80-94.

57. Soofi A, Levitan I, Dressler GR. Two novel EGFP insertion alleles reveal unique aspects of Pax 2 function in embryonic and adult kidneys. Dev Biol. 2012;365(1):241-250.

58. Harlow E, Lane D. Chapter 10: Cell Staining. In: Harlow E, Lane D. Antibodies: a laboratory manual. Cold Spring Harbor, New York, USA: Cold Spring Harbor Laboratory; 1988:359-420.

59. Soofi A, et al. The kielin/chordin-like protein (KCP) attenuates high-fat diet-induced obesity and metabolic syndrome in mice. J Biol Chem. 2017;292(22):9051-9062.

60. Soofi A, Zhang P, Dressler GR. Kielin/chordin-like protein attenuates both acute and chronic renal injury. J Am Soc Nephrol. 2013;24(6):897-905.

61. Lechner MS, Levitan I, Dressler GR. PTIP, a novel BRCT domain-containing protein interacts with Pax2 and is associated with active chromatin. Nucleic Acids Res. 2000;28(14):2741-2751. 